\title{
Palmas de comunidades ribereñas como recurso sustentable en la Amazonía brasileña
}

\author{
Palms of riverine communities as a sustainable resource in the Brazilian \\ Amazon
}

\author{
Ires Paula de Andrade Miranda, Edelcílio Marques Barbosa, Afonso Rabelo y \\ Filomena Ferreira Santiago
}

Botanical Research Coordination, Instituto Nacional de Pesquisas da Amazoni, AM, Brazil. Av. Andre Amazona, AM, Brazil. Av. Andre Araújo, 2936, Petropolis, CEP 69067-375, Manaus,AM-BRAZIL. Email Ires Paula de Andrade Miranda: ires@inpa.gov.br
Trabajo presentado al Simposio Internacional "LAS PALMERAS EN EL MARCO DE LA INVESTIGACIÓN PARA EL DESARROLLO EN AMERICA DEL SUR", del 07 a 09 de Noviem 2007 , . 07 al Museo de Historia Natural, Unir de San Marcos, Lima, Perú.

\section{Resumen}

En la Amazonía, el $30 \%$ de la población se encuentra representada por pequeños núcleos poblacionales aislados. Uno de los problemas más graves para el desarrollo de estas poblaciones es el relacionado con la disponibilidad de energía eléctrica, porque su ausencia o constante racionamiento no permiten la instalación de agro-industrias para el aprovechamiento de los recursos naturales,lo que podria proprocionar valor agregado de los productos regionales renovables. En consecuencia, la búsqueda por mejores condiciones socio-económicas tiene como única alternativa la extracción depredadora y rápida de las riquezas naturales, llevando a la destrucción acelerada de los ecosistemas. Las plantas oleaginosas, destinadas principalmente a la alimentación básica de la población, también pueden constituir la base de un modelo de desarrollo tecnológico industrial auto-sostenido, ya que presentan la ventaja de ser recursos en áreas antrópizadas de la Amazonia; y que posibilitan la retención del hombre en el campo y evitan con esto la degradación de los ecosistemas naturales. En el presente estudio se da a conocer el conjunto de actividades que facilitaron la determinación del potencial de biomasa del "huasaí" (Euterpe precatoria Mart.) y otras oleaginosas disponibles en comunidades ribereñas del Estado de Amazonas (Brasil); éstas actividades estuvieron asociadas a las posibilidades de su manejo sostenido para la generación de energía eléctrica. De esta forma, se presentan datos que sugieren condiciones del uso sostenible de la oferta de biomasa como fuente energética alternativa para desarrollar un programa de universalización de la energía eléctrica en la Amazonia brasileña.

Palabras clave: Palmas, desarrollo auto-sostenido, comunidades tradicionales, Euterpe precatoria, recursos naturales, Amazonía.

\section{Abstract}

In the Amazon, approximately $30 \%$ of the population is agglomerated in small villages or isolated areas. One of the most serious problems is the lack of electricity, where reduced supply and frequent rationing reduce life quality and prevent the instalation of industries that could raise the value of renewable regional products. Consequently, the pursuit of better socioeconomic conditions promote the quick depletion of natural resources, which invariably results in the accelerated destruction of local ecosystems. Oil palms, that are mainly used for basic nutrition of local populations, could also form the basis for models of self-sustained technological and industrial development. A quantitative survey of native species of oil palms offers an alternative for sustained development based on a technological and industrial model because this resource occurs in populated areas in the Amazon, which facilitates retaining the people in the field, preventing the depletion of natural ecosystems. This study presents the activities that were used to identify the potential of biomass of Euterpe precatoria Mart and other oil palms available in riverside communities in the state of Amazonas (Brazil). These activities are associated with the possibilities of using palm species for sustainable development of energy generation. It was possible to identify the conditions for a sustainable supply of biomass as an alternative energy source which contributes to the Energy Universalization Program in the Brazilian Amazon.

Keywords: Palm, sustainable, traditional community, Euterpe precatoria, Natural resources, Amazonia.

\section{Introducción}

Las áreas rurales de la Amazonia brasileña tienen un crecimiento desigual respecto a las grandes metrópolis amazónicas, debido a la ausencia de una economía regional organizada y con poca influencia en las decisiones político-económicas, de tal forma que el crecimiento de la renta per cápita de esas poblaciones es mínimo. Las poblaciones ribereñas, representadas por villas, pueblos, asentamientos pequeños y núcleos poblacionales están generalmente aisladas de las grandes ciudades amazónicas en su mayoría por la ausencia de vías de comunicación rápidas y continuas; provocando situaciones económicas y culturales desfavorables. En consecuencia; en la búsqueda por mejores condiciones socio-económicas, la alternativa inmediata es la explotación desmesurada y rápida de las riquezas naturales, con la consecuente acelerada destrucción de los ecosistemas locales. Una de las posibles alternativas para minimizar la destrucción de los ecosistemas locales, sería promover la formación de cooperativas; estimulando la inversión en eco-industrias integradas dentro de una cadena de producción, que contemple las demandas principalmente de la comunidad local, con el aprovechamiento racional de la biodiversidad.

En áreas amazónicas periódicamente inundadas, existen áreas desmatadas y pastos abandonados, con baja diversidad de palmeras pero con abundancia de otras especies (Bondar, 1964; Galeano, 1992; Kahn \& Granville, 1992; Anderson et al., 1985; Anderson, 1988; Moussa et al., 1994; Henderson, 1995; Ribeiro, 1999; Miranda et al., 2001; Miranda et al., 2003; Miranda \& Rabelo, 2006). En la región amazónica, las palmeras deben ser consideradas como especies prioritarias en la evaluación de productos forestales no madereros, por los múltiples usos que las comunidades rurales y nativas pueden darles (Strudwick \& Sobel, 1988 y Bernal, 1992) y que pueden constituir la base para la aplicación de un modelo de desarrollo tecnológico-industrial auto-sostenido.

Considerando el estudio de palmeras promisorias, el presente trabajo enfatiza sobre la palmera "huasai" (Euterpe precatoria Mart.), especie predominante, con gran densidad poblacional, en 


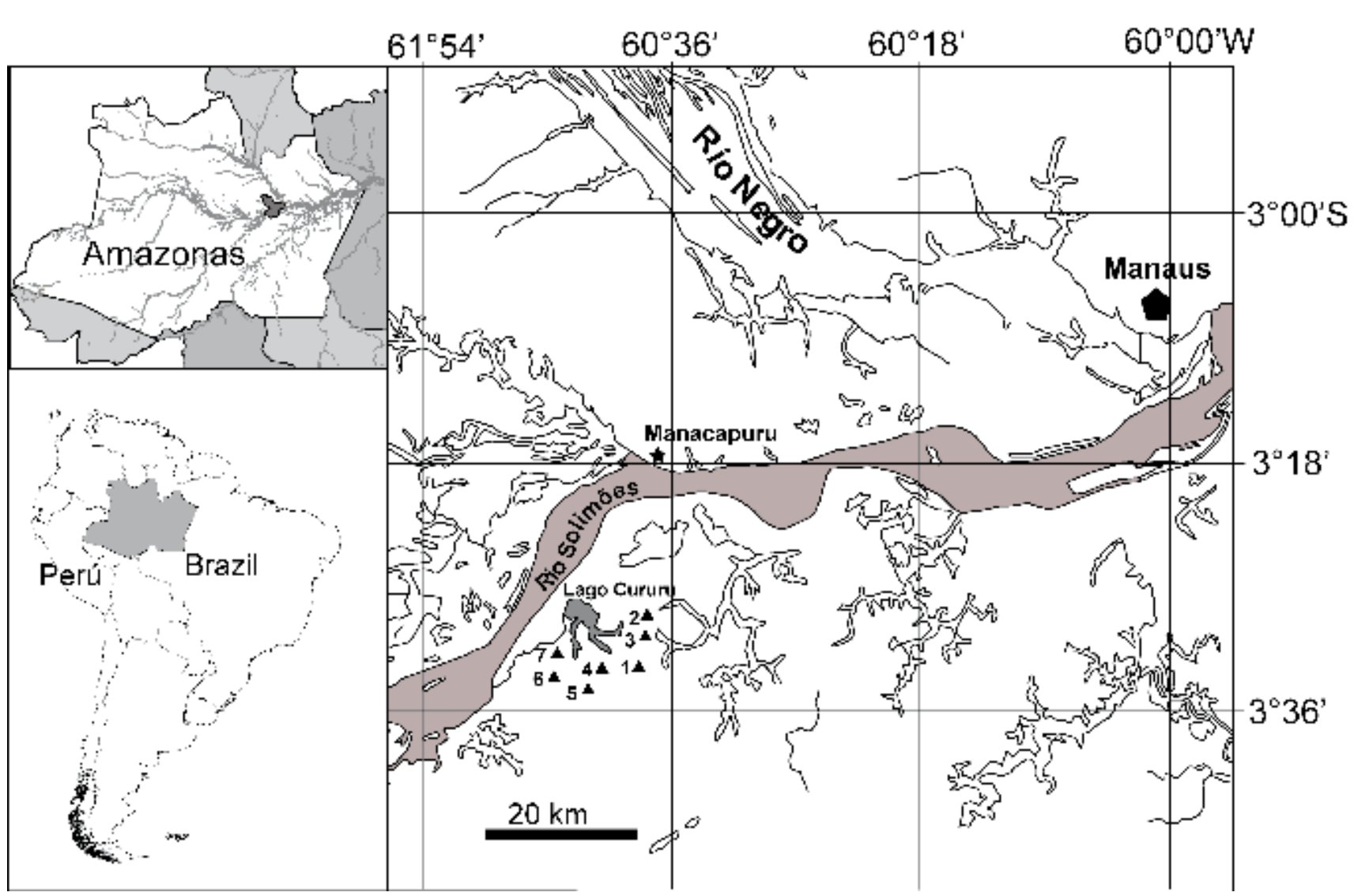

Figura 1. Mapa de las localidades evaluadas en la cuenca del rio Solimões: $1=$ Cordeiro, $2=$ Tierra Negra1, $3=$ Tierra Negra2, $4=$ Divino Espírito Santo1, 5 = Divino Espírito Santo2, 6 = Buen Jesus Cururu, 7 = Ciudad Nueva Cururu, LC = Lago de Cururu.

áreas de floresta de tierra firme y en áreas inundables de la Amazonía (Kahn \& Henderson, 1999). El "huasaî" es una palmera monocaule, de aproximadamente $20 \mathrm{~m}$ de altura, de tallo liso con $25 \mathrm{~cm}$ de diámetro; hojas pinnadas con $6 \mathrm{~m}$ de largo, vaina cerrada y lisa, de coloración verde con 1,5 m de largo, peciolo corto; pinnas pendientes y dispuestas regularmente. Inflorescencia intrafoliar en la antesis, frutos globosos lisos, mediendo $1,1 \times 1,1 \mathrm{~cm}$ de diámetro, coloración negro-violácea cuando maduro (Miranda \& Rabelo, 2006). Los frutos del "huasaî", pueden ser utilizados en la producción de vinos, helados, jugos, dulces, etc. (Miranda \& Rabelo, 2008). El tallo, bastante resistente, es utilizado en la construcción de paredes de casas rurales y el palmito (chonta) es de excelente calidad alimenticia. Después de la utilización de la pulpa del fruto, las semillas pueden ser utilizadas como fuente de biomasa para la generación de energía eléctrica sin comprometer el ecosistema; fortaleciendo de esta manera la cadena productiva y el desarrollo eco-sostenido de las poblaciones ribereńas. A pesar de las grandes densidades poblacionales del "huasaî" su uso en escala agro-industrial presenta enormes dificultades debido a la falta de políticas económicas y el interés de los sectores industriales, académicos y gubernamentales.

El presente trabajo estima el número y la distribución estructural de las poblaciones de "huasai" por unidad de área, en las localidades rurales del Municipio de Manacapuru (Amazonas, Brasil) y sugiere el uso de la biomasa disponible de "huasai" ( $E$. precatoria) como fuente de generación de energía eléctrica.

\section{Área de estudio}

La cuenca del río Amazonas es una región muy extensa, de aproximadamente $6000000 \mathrm{~km}^{2}$, siendo mayor que varios países del mundo.

Tabla 1. Ubicación de los lugares de muestreo en la cuenca del rio Solimões, municipio de Manacapuru, de Amazonas, Brasil.

\begin{tabular}{lccccc}
\hline Comunidad (Localidad) & $\begin{array}{c}\text { Unidad de } \\
\text { vegetación }\end{array}$ & Transectos & Altitud (m) & Coordenadas \\
\hline & & & & Sur & Oeste \\
Cordeiro (área 1) & Floresta & 01 & 31 & -3.5604 & -60.6528 \\
Tierra Negra1 (área 2) & & 01 & 32 & -3.4886 & -60.6410 \\
Tierra Negra2 (área 3) & Floresta & 01 & 32 & -3.4944 & -60.6379 \\
Divino Espírito Santo1 (área 4) & & 01 & 36 & -3.5587 & -60.6885 \\
Divino Espírito Santo2 (área 5) & Floresta & 01 & 37 & -3.5645 & -60.6908 \\
Buen Jesus de Cururu (área 6) & Floresta & 01 & 35 & -3.5702 & -60.7472 \\
Ciudad Nueva de Cururu (área 7) & Floresta & 01 & 34 & -3.5589 & -60.7486 \\
\hline
\end{tabular}


Tabla 2. Total de los individuos de huasaí (Euterpe precatoria) en el levantamiento, árbol con racimo y total de los racimos en diferentes localidades en el Lago Cururu, municipio de Manacapuru, estado de Amazonas, Brasil.

\begin{tabular}{|c|c|c|c|c|}
\hline Comunidad (Localidad) & $\begin{array}{c}\text { Total de } \\
\text { individuos (ha) }\end{array}$ & $\begin{array}{l}\text { № Individuos con } \\
\text { racimo (ha) }\end{array}$ & $\begin{array}{r}\text { Total de los } \\
\text { racimos (ha) }\end{array}$ & Área de los levantamientos \\
\hline Cordeiro (área 1) & 124 & 56 & 80 & 1 hectárea $10 \times 1000 \mathrm{~m}$ \\
\hline Tierra Negra(1) (área 2) & 91 & 26 & 35 & 1 hectárea $20 \times 500 \mathrm{~m}$ \\
\hline Tierra Negra(2) (área 3) & 207 & 94 & 153 & 1 hectárea 10x1000 m \\
\hline Divino Espírito Santo1 (área 4) & 214 & 79 & 97 & 1 hectárea 10x1000 m \\
\hline Divino Espírito Santo2 (área 5) & 226 & 103 & 183 & 1 hectárea 10x1000 m \\
\hline Bom Jesus de Cururu (área 6) & 221 & 121 & 181 & 1 hectárea 10x1000 m \\
\hline Ciudad Nueva de Cururu (área 7) & 110 & 42 & 56 & 1 hectárea 10x1000 m \\
\hline Suma total & 1193 & 521 & 785 & 7 hectárea \\
\hline Promedio & 170,43 & 74,43 & 112,14 & 1 hectárea \\
\hline
\end{tabular}

El Municipio de Manacapuru está situado en la margen izquierda del Rio Solimóes (región central del Rio Amazonas) con $7329 \mathrm{Km}^{2}$. Presenta una amplia red de hidrovías con varios lagos, de los cuales se destaca el lago de Cururu (Fig. 1). Siete comunidades rurales (tabla 1) fueron evaluadas en el periodo 2005-2006. La vegetación es de floresta natural, con árboles altos de $35 \mathrm{~m}$ de altura en media, encontrándose epífitas; como bromélias, orquídeas y hongos. El suelo es relativamente plano, con zonas arenosas y arcillosas en las márgenes del lago.

Comunidad de Cordeiro: se ubica en la margen derecha al borde del río Solimóes, en el ramal central del lago de $\mathrm{Cu}$ ruru.

Comunidades de Tierra Negra 1 y 2: están ubicadas en la margen derecha y al borde del río Solimóes, en el ramal derecho del lago de Cururu. Las características edáficas y forestales son similares a las descritas en la Comunidad de Cordeiro.

Comunidades Divino Espírito Santo 1 y 2: estan ubicadas en la margen derecha y al borde del río Solimóes, en el ramal izquierdo del lago de Cururu. Las características edáficas y forestales son similares a las descritas en la Comunidad de Cordeiro.
Comunidad de Buen Jesús de Cururu: estan ubicadas en la margen derecha y al borde del río Solimóes, en el ramal izquierdo del lago de Cururu. Las características edáficas y forestales son similares a las descritas en la Comunidad de Cordeiro.

Comunidad de Cidade Nova de Cururu: se ubica en la margen derecha y al borde del río Solimóes, en el ramal izquierdo del lago de Cururu. Las características edáficas y forestales son similares a las descritas en la Comunidad de Cordeiro.

\section{Material y métodos}

La densidad poblacional de especímenes adultos de E. precatoria Mart. fue evaluada en cada localidad a partir del método de transectos, midiendo de $10 \times 1000 \mathrm{~m}$, con 20 parcelas de 10 x $50 \mathrm{~m}$. Paralelamente, fueron realizados perfiles esquemáticos de los individuos con su respectiva identificación (Fig. 2).

En los análisis biométricos de los frutos fue utilizado un vernier digital y balanza analítica y para el cálculo de la materia seca una estufa con ventilación con temperatura mayor de $100{ }^{\circ} \mathrm{C}$.

Las siguientes variables fueron analizadas: masa media de los racimos $(\mathrm{kg})$, descontando el peso del la raquis principal y de las raquillas, diámetro del fruto $(\mathrm{cm})$, masa media del fruto (g), que fue calculada en tres muestras de 25 frutos por racimo

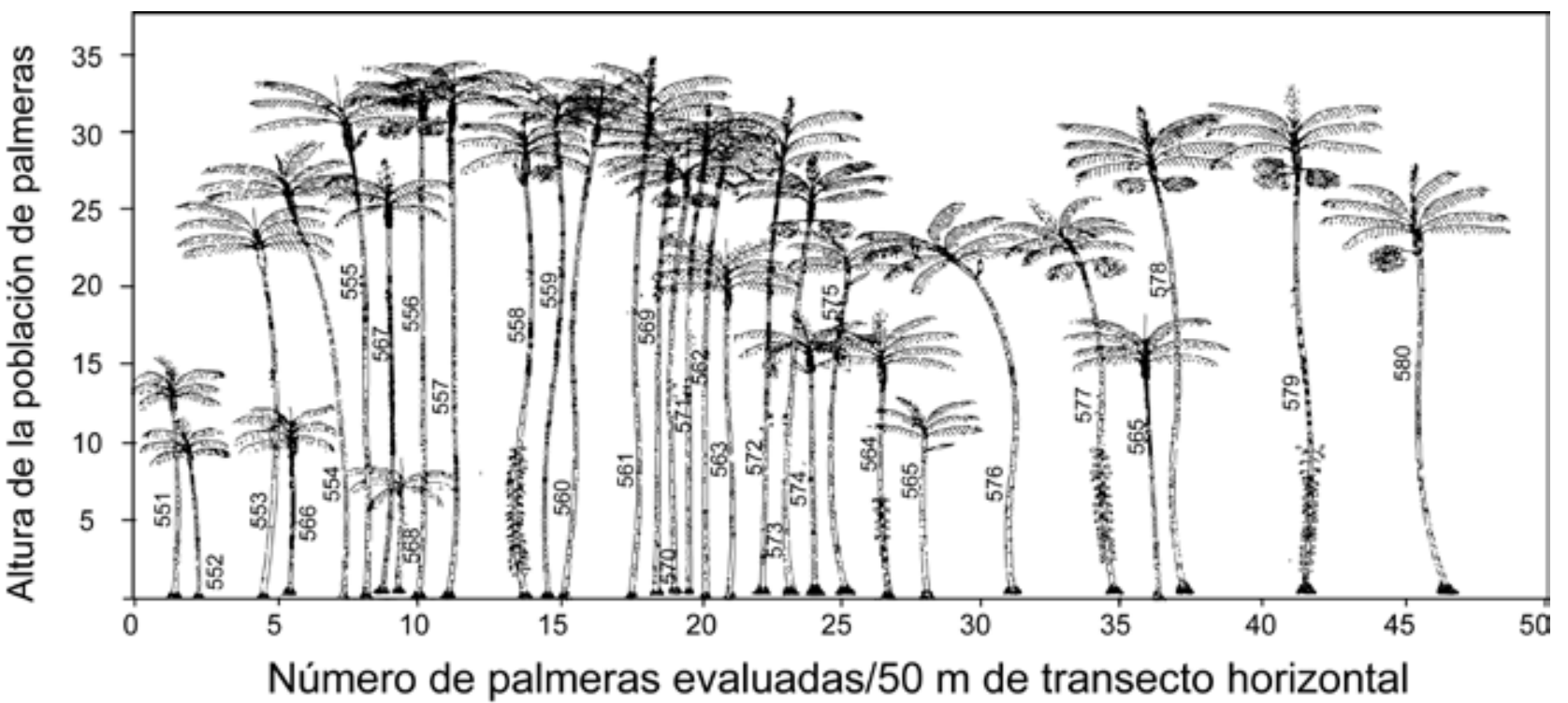

Figura 2. Perfiles de la vegetación con árbol palmera huasaí en lugares de muestreo en el municipio de Manacapuru, estado de Amazonas, Brasil. 
Tabla 3. Estimación de la productividad $\left(\mathrm{t} \mathrm{ha}^{-1} \mathrm{año}^{-1}\right)$ de huasaí (Euterpe precatoria) sometidas a las diferentes densidades y sistemas de levantamiento, en el lago Cururu, municipio de Manacapuru, estado de Amazonas, Brasil(1).

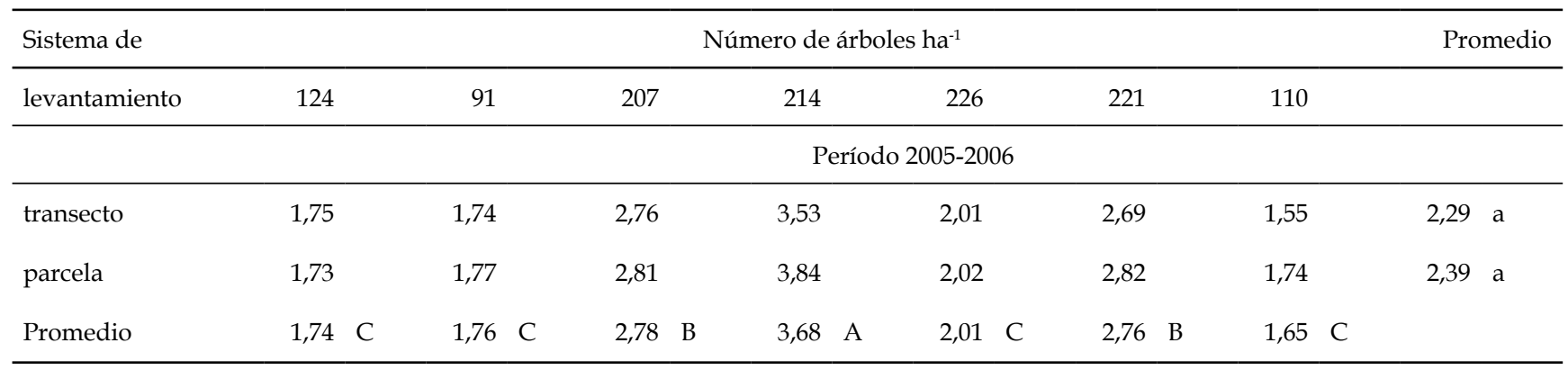

(1) En el período 2005/2006, Promedio de la misma letra, mayúscula en la línea y minúscula en la columna, no difieren entre sí por la prueba de Tukey a $5 \%$ de probabilidad.

y número total de frutos por racimo. La producción estimada por hectárea t.ha ${ }^{-1}$ en el periodo 2005-2006 fue calculada multiplicándose la masa del fruto por racimo por el número total de plantas por hectárea.

Los datos colectados fueron analizados por análisis de varianza (test F) y la significancia de las medias fueron comparadas con el test de Tukey con 5\% de probabilidad (Tabla 3).

Al término del periodo 2005-2006, fueron determinados el número de frutos producidos durante un año, la variación de la masa del fruto por racimo según el mes de la cosecha y la productividad estimada en t.ha ${ }^{-1}$. año ${ }^{-1}$. Por tanto, la productividad fue determinada, utilizando la fórmula de Robinson \& Nel (1988):

t.ha" año $^{-1}=$ [producción $\left(\right.$ t.ha $\left.{ }^{-1}\right) /$ duración del ciclo (meses) $]$ x 12 .

\section{Resultados}

En función de la productividad del "huasâ’", las áreas más productivas fueron las localidades de las comunidades de Tierra

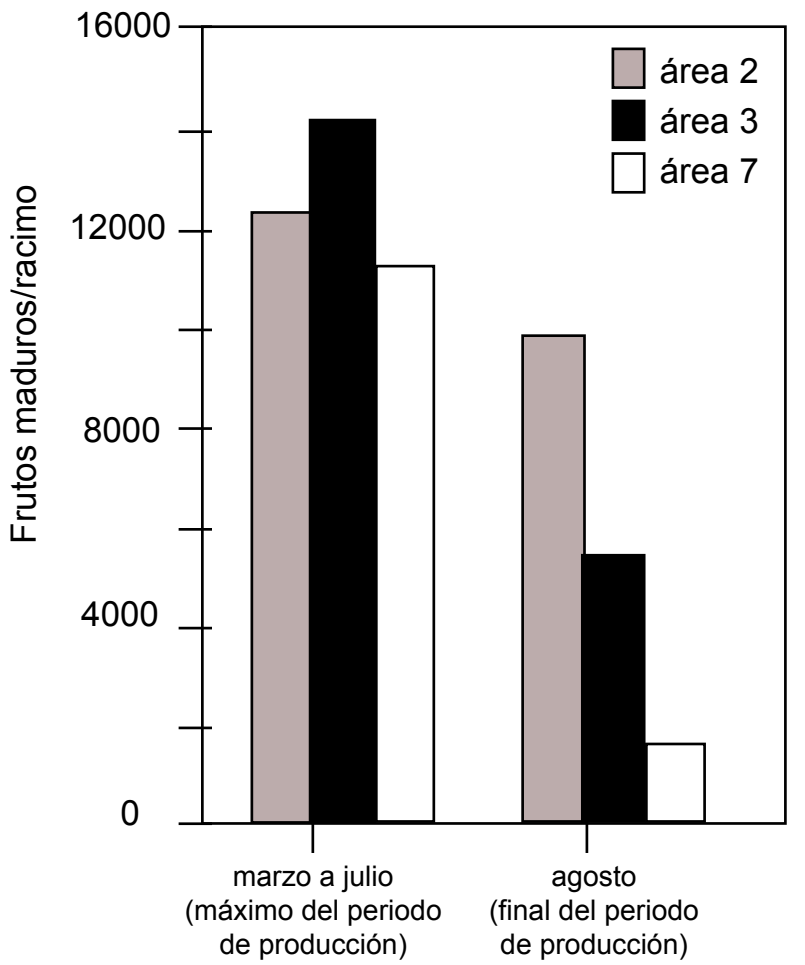

Figura 3. Promedio de los frutos maduros por racimo de huasaí ( $E u-$ terpe precatoria) en las localidades más productivas de los lugares de muestreo en el municipio de Manacapuru, estado de Amazonas, Brasil.
Negra 1 (área 2), Tierra Negra 2 (área 3) y Ciudad Nueva de Cururu (área 7) (Tabla 2 y Fig. 3). Se observó también que la mayor producción de racimos fue durante los meses de marzo a julio, período de aumento del volumen de los ríos, como también un pico menor de producción de frutos a fines de agosto. La colecta de los frutos fue realizada por 2-4 personas y la producción fue de 1 a 4 racimos por planta. Siete racimos equivalen en promedio a $45 \mathrm{Kg}$. También determinamos que para el funcionamiento de un generador de energía eléctrica de $50 \mathrm{Kw}$ sería necesario 365 hectáreas con aproximadamente 90 plantas productivas por hectárea. Fueron encontradas otras especies asociadas a E. precatoria en las áreas de estudio: Attalea phalerata, Astrocaryum murumuru, Astrocaryum aculeatum y Attalea phalerata.

El promedio de la densidad poblacional de E. precatoria encontrada en el presente estudio fue de 170 individuos/ha, correspondiendo a 1,8 toneladas de frutos por hectárea en las áreas inundables (Tabla 2 y Fig. 4).

En cada parcela fueron evaluados los siguientes parámetros: número de individuos por hectárea, número de racimos por planta y número de frutos por racimo (Tabla 2).

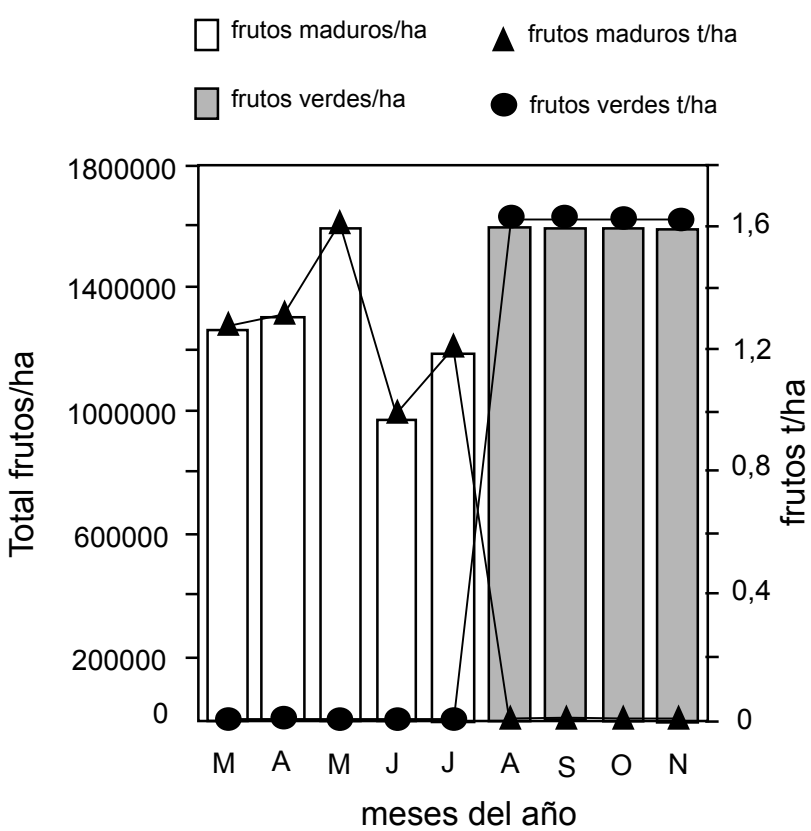

Figura 4. Total de los frutos y producción de huasaí (Euterpe precatoria) en el periodo 2005/2006 en tonelada por hectárea durante los meses de estudio en el municipio de Manacapuru, estado de Amazonas, Brasil. 


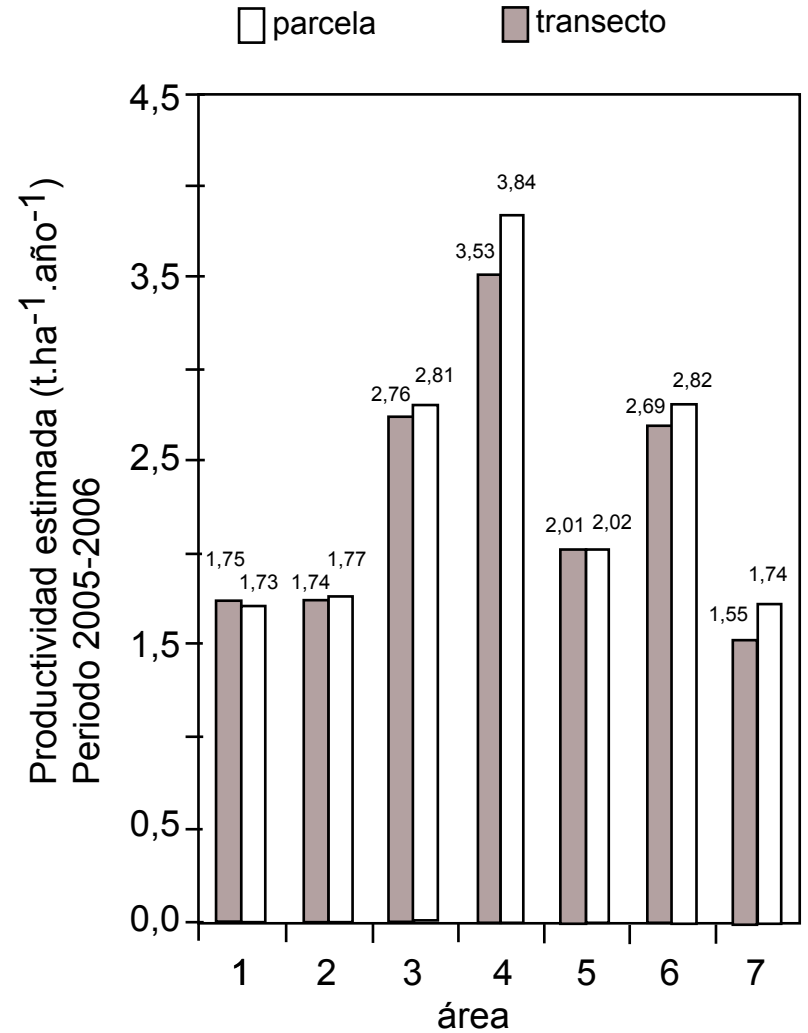

Figura 5. Estimación de la productividad de huasaí (Euterpe precatoria) en el periodo 2005/2006 por localidad de muestreo en el município de Manacapuru, estado de Amazonas, Brasil.

La productividad fue calculada utilizando parámetros de estimación de acuerdo con los datos colectados de algunas áreas estudiadas. El aumento de la densidad del "huasaî" que podría llegar hasta cerca de 4 toneladas por hectárea/año; fue comparado con el periodo 2005/2006. De modo general, con relación a la masa del racimo evaluada en la densidad encontrada en una población de 214 plantas (número de plantas ha $\mathrm{a}^{-1}$ ) fue de $14 \%$ a $20 \%$ inferior a la observada en las densidades 124, 207 y 110. La masa del fruto por racimo observada tuvo comportamiento variado e intermediario, diferenciándose algunas veces del área más densa (Tabla 3 y Fig. 5), con dependencia del aumento del nivel de las aguas.

\section{Discusión}

Según Filho \& Klunge (2001), la proximidad entre las plantas puede provocar aumento en la altura de ellas, debido a la competencia por la luz solar, provocando mayor demanda de agua, nutrientes y reguladores vegetales, y como consecuencia puede afectar la cantidad y calidad de los frutos.

La densidad media de 170 plantas por hectárea, permite una productividad suficiente para potencializar la cadena productiva del "huasaî" en las áreas ribereńas del Estado de Amazonas. Las densidades observadas corresponden a los datos de Kahn \& Granville (1992), Castro (1993), Kahn \& Henderson (1999) y Rocha (2004).

Los resultados demuestran que existe una producción de 1,8 toneladas de frutos por hectárea, y es posible afirmar que el "huasaî", en las comunidades ribereñas estudiadas, presenta una alta productividad durante el periodo de creciente (del nivel de las aguas); contribuyendo en su aprovechamiento económico dentro de la cadena productiva agro-industrial.
Nuestros resultados sugieren que durante el periodo de mayor productividad del "huasai", se establezcan programas socioeconómicos continuos (permanencia del campesino en las áreas rurales, programas educacionales, generación de agro-industrias, etc.) que puedan incentivar un mejor uso del "huasaî", con el objetivo de obtener de este producto natural, mayor provecho económico, lo cual redundaría en una mejor calidad de vida del hombre amazónico.

\section{Agradecimientos}

Esta investigación fue financiada gracias a fondos obtenidos a través del proyecto NERAM/CNPq, cordinado por el Dr. Rubem Cesar Rodrigues. A los Drs. Francis Kahn (IRD) y Betty Millán y a su equipo (UNMSM) por la oportunidad de mi participación en el Simposio Internacional "Las Palmeras en el Marco de la Investigación para el Desarrollo en América del Sur" realizado en Lima, Perú. A los investigadores Luis Lópes Lozano, Pedro Mera y Gina Frausin por su apoyo en la traducción y transcripción en espańol. A Thiago Andrade por la traducción del resumen en inglés. A Felipe França de Moraes por su asistencia en la elaboración de las figuras que ilustran el trabajo. Un agradecimiento muy especial a las personas que han colaborado en el trabajo de campo.

\section{Literatura citada}

Anderson A.B. 1988. Use and management of native forests dominated by açai palm (Euterpe precatoria Mart.) in the Amazon estuary. Advances in Economic Botany 6: 144-154.

Anderson A.B., A.Gély, J. Strudwick, G.L. Sobel \& M.G.C. Pinto. 1985. Um sistema agroflorestal na várzea do estuário amazônico (Ilha das Onças, Municipio de Barcarena, Estado doPará). Acta Amazonica 15 suppl.: 195-224.

Bernal R.G. 1992. Colombian palm products. In: M. Plotkin, L. Famolare, Eds. Sustainable harvest na marketing of rain forest products. Washington: Island Press/Conservation International. $325 \mathrm{p}$.

Bondar G. 1964. Palmeiras do Brasil. Instituto de Botânica, São Paulo. Pp. 5-159.

Castro A. 1993. Extractive exploitation of the açaí near Manaus, Amazônia. In : Food and Nutrition in the tropical forest: Biocultural interactions, Hladik CM et al (eds), Man and the Biosphere series, Vol. 15 Parthenon Publ Group, pp. 779-782.

Filho J.A.S. \& R.A. Kluge. 2001. Produção da bananeira 'Nanicão' em diferentes densidades de plantas e sistemas de espaçamento. Brasília. Pesq. agropec. bras. 36(1):105-113.

Galeano G. 1992. Las palmas de la region de Araracuara. TROPENBOS, Colômbia. 180 p.

Henderson A. 1995. The palms of the Amazon. Oxford University Press, New York. 362 p.

Kahn F. \& J. De Granville. 1992. Palms in forest ecosystems of Amazonia. U.S.A, Springer-Verlag Berlin Heidelberg, 226p.

Kahn F. \& A. Henderson. 1999. An Overview of the Palm of the Várzea in the Amazon Region. In: C. Padoch, J.M. Ayres, M. Pinedo-Vasquez and H. Henderson, Eds. Várzea diversity, development, and conservation of Amazonia's whiteuwater floodplains. The New York Botanical Gardens. New York. Pp. 187-193.

Miranda I.P.A., A. Rabelo, C.R. Bueno, E.M. Barbosa \& M.N.S. Ribeiro. 2001. Frutos de palmeiras da Amazônia. MCT/ INPA. Manaus. 120p.

Miranda I.P.A., E.M. Barbosa, J.L.B. Guillaumet, M.R.L. Rodrigues \& M.F.F. Silva (eds.) 2003. Ecossistemas florestais em áreas manejadas na Amazônia. MCT/INPA/PPG-7. Manaus-AM. 305p. 
Miranda I.P.A. \& A. Rabelo. 2006. Guia de Identificação das Palmeiras de um Fragmento Florestal Urbano de Manaus. EDUA/INPA. Manaus. 228p.

Miranda I.P.A \& A. Rabelo. 2008. Guia de Identificação das Palmeiras de Porto Trombetas, Oriximiná-PA. EDUA/INPA. Manaus, 365 p. ilust.

Moussa F., I.P.A. Miranda \& F. Kahn, 1994. Palmeiras no herbário do INPA, INPA/ORSTOM. Manaus, Brasil, 93 p.

Ribeiro J.E.L.S., M.J.G. Hopkins, A. Vicentini, C.A. Sothers, M.A.S. Costa \& et al. 1999. Flora da Reserva Ducke: Guia de identificação das plantas vasculares de uma floresta de terra-firme na Amazônia central. Manaus:INPA. 816 p.
Robinson J.C. \& D.J. Nel. 1988. Plant density studies with banana (cv. Willians) in a subtropical climate. I. Vegetative morphology, phenology and plantation microclimate. Ashford. Journal of Horticultural Science 63(2):303-313.

Rocha E. 2004. Potencial ecológico para o manejo de frutos de açaizeiro (Euterpe precatoria Mart.) em áreas extrativistas no Acre, Brasil. Acta Amazonica 34(2):237-250.

Strudwick J. \& G.L. Sobel. 1988. Uses of Euterpe oleracea Mart. in the Amazon estuary, Brazil. Adv. Econ. Bot. 6 : 225253. 